\title{
Pre-hospital, in-hospital and post-hospital factors associated with sleep quality among COVID-19 survivors 6 months after hospital discharge: cross-sectional survey in five cities in China
}

Leiwen Fu*, Yuan Fang*, Dan Luo*, Bingyi Wang* , Xin Xiao, Yuqing Hu, Niu Ju, Weiran Zheng, Hui Xu, Xue Yang, Paul Shing Fong Chan, Zhijie Xu, Ping Chen, Jiaoling He, Hongqiong Zhu, Huiwen Tang, Dixi Huang, Zhongsi Hong, Xiaojun Ma, Yanrong Hao, Lianying Cai, Jianrong Yang, Shupei Ye, Jianhui Yuan, Yao-Qing Chen, Fei Xiaot, Zixin Wangt and Huachun Zout

\section{Background}

Understanding factors associated with post-discharge sleep quality among COVID-19 survivors is important for intervention development.

\section{Aims}

This study investigated sleep quality and its correlates among COVID-19 patients 6 months after their most recent hospital discharge.

\section{Method}

Healthcare providers at hospitals located in five different Chinese cities contacted adult COVID-19 patients discharged between 1 February and 30 March 2020. A total of 199 eligible patients provided verbal informed consent and completed the interview. Using score on the single-item Sleep Quality Scale as the dependent variable, multiple linear regression models were fitted.

\section{Results}

Among all participants, $10.1 \%$ reported terrible or poor sleep quality, and $26.6 \%$ reported fair sleep quality, $26.1 \%$ reported worse sleep quality when comparing their current status with the time before COVID-19, and 33.7\% were bothered by a sleeping disorder in the past 2 weeks. After adjusting for significant background characteristics, factors associated with sleep quality included witnessing the suffering (adjusted $B=-1.15,95 \%$ $\mathrm{Cl}=-1.70,-0.33$ ) or death (adjusted $\mathrm{B}=-1.55,95 \% \mathrm{Cl}=-2.62$, -0.49) of other COVID-19 patients during hospital stay, depressive symptoms (adjusted $\mathrm{B}=-0.26,95 \% \mathrm{Cl}=-0.31,-0.20$ ), anXiety symptoms (adjusted $\mathrm{B}=-0.25,95 \% \mathrm{Cl}=-0.33,-0.17$ ), posttraumatic stress disorders (adjusted $\mathrm{B}=-0.16,95 \% \mathrm{Cl}=-0.22$, -0.10 ) and social support (adjusted $\mathrm{B}=0.07,95 \% \mathrm{Cl}=0.04,0.10$ )

\section{Conclusions}

COVID-19 survivors reported poor sleep quality. Interventions and support services to improve sleep quality should be provided to COVID-19 survivors during their hospital stay and after hospital discharge.

\section{Keywords}

COVID-19 survivors; sleep quality; pre-hospital factors; in-hospital factors; post-hospital factors; China.

\section{Copyright and usage}

(C) The Author(s), 2021. Published by Cambridge University Press on behalf of the Royal College of Psychiatrists. This is an Open Access article, distributed under the terms of the Creative Commons Attribution licence (https://creativecommons.org/ licenses/by/4.0/), which permits unrestricted re-use, distribution, and reproduction in any medium, provided the original work is properly cited.
Good sleep is part of good quality of life. High-quality and efficient sleep of adequate duration helps to consolidate memory, regulate the immune system and coordinate neuroendocrine functions. ${ }^{1}$ By contrast, poor sleep quality leads to a broad range of adverse outcomes, including cardiovascular diseases, poor mental health status, cognitive impairment and even overall mortality., ${ }^{2,3}$

COVID-19 may negatively affect sleep quality and other mental health outcomes among patients admitted to hospital, patients with mild and asymptomatic cases, and survivors. ${ }^{4-8}$ COVID-19 symptoms and experience during hospital stay are stressors affecting mental health status. ${ }^{4}$ Moreover, treatment for COVID-19 may have adverse effects on mental health and contribute to insomnia. ${ }^{9}$ Furthermore, SARS-Cov-2 might also infect the brain, which could directly cause adverse effects on brain functions and mental health. ${ }^{5}$

Understanding factors associated with sleep quality among COVID-19 survivors after hospital discharge is important for intervention development and service planning to enhance well-being.

\footnotetext{
* Joint first authors.

† Co-corresponding authors

*The original version of this article was published with an error in an author's name. A notice detailing this has been published and the erro rectified in the online PDF and HTML copies.
}

Cross-sectional studies showed that $29.5 \%$ and $26 \%$ of COVID-19 survivors in Wuhan, China, were bothered by a sleeping disorder 1 month and 6 months after discharge, respectively (measured by a single item within the Patient Health Questionnaire (PHQ-9): 'Whether you have trouble falling or staying asleep, or sleeping too much in the past two weeks' and a self-reported symptom questionnaire: 'What do you think about your sleeping compared with the status prior to COVID-19'). ${ }^{6}$ A cohort study reported that $26 \%$ of COVID-19 survivors suffered from sleep difficulties 6 months after hospital discharge. ${ }^{10}$ Moreover, 40\% of COVID-19 survivors in Italy reported insomnia (as measured by the Women's Health Initiative Insomnia Rating Scale). ${ }^{7}$ Only a limited range of factors associated with sleep quality have been reported. Being female, younger and having a history of psychiatric disorder were associated with poor sleep quality. ${ }^{7}$ Previous studies showed that sleep problems among survivors of severe acute respiratory syndrome (SARS) were likely to be persistent; $47 \%$, $50 \%$ and $44 \%$ of survivors reported difficulty sleeping 3,6 and 12 months after hospital discharge, respectively. ${ }^{11}$

A systematic review suggested that there were three groups of risk factors for sleep quality among patients after hospital admission and/or critical illness: pre-hospital factors, in-hospital factors and post-hospital factors. ${ }^{12}$ Pre-hospital factors included pre-hospital 
sleep quality, self-reported concurrent diseases before hospital admission and smoking. ${ }^{12}$ Disease severity at hospital admission, ventilation days, intensive care unit (ICU) admission and length of hospital stay were in-hospital risk factors for sleep quality after hospital discharge. ${ }^{12}$ The main post-hospital factors associated with sleep quality were depression, anxiety and stress. ${ }^{12}$ These factors were considered in this study.

In addition, COVID-19 survivors may have experienced multiple stressors and traumatic events, such as having family members become infected with and/or die of COVID-19, and witnessing the painful symptoms and/or death of other patients. ${ }^{4}$ Corticosteroid therapy is beneficial for modulating the host inflammatory response to COVID-19. ${ }^{9}$ However, sleep disturbance is a commonly cited adverse effect of corticosteroid. ${ }^{13}$ The Chinese government has been providing psychological support for COVID-19 patients and their family members, as well as for people under quarantine, since 8 March $2020 .{ }^{14}$ Screening, prevention and treatment for mental health problems have been pro-actively provided to COVID-19 patients during their hospital stay. ${ }^{14}$ COVID-19 survivors are also encouraged to seek psychological support after hospital discharge. Community healthcare centres are coordinating with psychotherapists and social workers to provide such services to COVID-19 survivors in need. ${ }^{14}$ This study also investigated the association between receiving mental health support services during hospital stay and/or after hospital discharge with post-discharge sleep quality among COVID-19 survivors. Moreover, one study evaluating psychological interventions targeting COVID-19 patients showed that better social support predicted improvement in sleep quality. ${ }^{15}$ Therefore, social support after hospital discharge was also considered by this study. Furthermore, about $7-8 \%$ of COVID-19 survivors tested positive again after hospital discharge. ${ }^{16-18}$ However, there was no consensus about the cause of such phenomena. ${ }^{19,20}$ Positive retest results may be another stressor for COVID-19 survivors.

There is a dearth of studies investigating the longer-term impact of COVID-19 on sleep quality. To address these gaps, this study investigated sleep quality among COVID-19 patients 6 months after their most recent hospital discharge. Factors associated with sleep quality were also investigated, including background characteristics and pre-hospital, in-hospital and post-hospital factors.

\section{Method}

\section{Study design}

This was a cross-sectional telephone survey among 199 COVID-19 patients 6 months after their hospital discharge. The study was conducted during August to September 2020.

\section{Participants and data collection}

Participants were recovered adult COVID-19 patients discharged between 1 February and 30 March 2020. The conveniently selected study sites included five hospitals located in five Chinese cities (i.e., Wuhan, Shenzhen, Zhuhai, Dongguan and Nanning) with high, moderate and low COVID-19 case-load. Wuhan is the capital city of Hubei Province, which is most affected by the COVID-19 pandemic in China. Shenzhen, Zhuhai and Dongguan are cities in Guangdong Province, which has the second largest number of COVID-19 cases in China. Nanning is the capital city of Guangxi Province, which has been less affected by the COVID-19 pandemic. As of 9 November 2020, the numbers of recovered COVID-19 patients in these Chinese cities were 46475 in Wuhan, 72 in Nanning, 468 in Shenzhen, 109 in Zhuhai and 100 in Dongguan. ${ }^{21}$ COVID-19 survivors who were under 18 years old were excluded for the following reasons. First, the measurement of sleep quality (single-item Sleep Quality Scale; SQS) was not validated in a population under 18 years old. ${ }^{22}$ No study used this scale to measure sleep quality among children or adolescents. Second, most of the patients under 18 years old in the records kept by the collaborative hospitals were very young (5-10 years old). It would have been difficult for them to answer our questionnaire, which used relatively complicated psychosocial measurements. Asking their parents to answer the questionnaire would create additional bias.

According to the treatment guidelines in China, recovered COVID-19 patients should be quarantined for 14 days in designated facilities, followed by another 14-day home-based quarantine after hospital discharge. Their contact information is kept by the hospitals for follow-up assessments and services. Recruitment was facilitated by medical staff in the five participating hospitals, who were responsible for follow-up visits and services for recovered COVID-19 patients after hospital discharge. They contacted all discharged COVID-19 patients discharged between 1 February and 30 March 2020 who were listed in their registries. Verbal informed consent was obtained from all participants. Verbal consent was witnessed and formally recorded. The aforementioned medical staff screened prospective participants' eligibility to join the study, briefed them about the study information and invited them to complete a telephone-based interview. Upon appointment, trained interviewers confirmed participants' informed consent and conducted the telephone-based interview. Participants were assured that identifiable information would be kept confidential, and that withdrawal from the study would not affect their right to use any treatment services. The interview lasted for about $35 \mathrm{~min}$. No incentive was given to the participants. The authors assert that all procedures contributing to this work comply with the ethical standards of the relevant national and institutional committees on human experimentation and with the Helsinki Declaration of 1975, as revised in 2008. All procedures involving human subjects were approved by the Sun Yat-sen University (Shenzhen) (ref. 2020031).

Of the 317 recovered COVID-19 patients discharged from these hospitals, 27 were under 18 years old (the scale used in this study was not applicable to these patients), 22 had changed their telephone number and one had died in a car accident. The remaining 267 eligible patients were contacted by the research team; 68 eligible patients refused to participate in the study. A total of 199 eligible patients provided consent and completed the telephone-based interview. The response rate was $74.5 \%$ (Wuhan: $31 / 49,63.3 \%$; Nanning: 56/72, 77.8\%; Shenzhen: 38/50, 76.0\%; Zhuhai: 39/51, 76.5\%; and Dongguan: $35 / 45,77.8 \%)$.

\section{Measures}

Development of the questionnaire

A panel consisting of one epidemiologist, two public health researchers, a health psychologist and a clinician was formed to develop the questionnaire used in the current study.

\section{Background characteristics}

Participants were asked to report on sociodemographic characteristics, including age, gender, permanent residency status, highest educational level, relationship status, monthly personal income, employment status and whether they had children.

\section{Sleep quality}

A single-item SQS was used to measure global sleep quality over a 7 day recall period with a rating ranging from 0 to 10 . A higher score 
indicated better sleep quality. ${ }^{22}$ Cut-off scores of $1,4,7$, and 10 were used to define poor, fair, good and excellent sleep quality, respectively. ${ }^{22}$ The single-item SQS uses more suitable measurement characteristics to assess sleep quality compared with lengthier sleep questionnaires such as the Pittsburgh Sleep Quality Index (PSQI) and the Morning Questionnaire-insomnia. ${ }^{22}$ This instrument has been demonstrated to be a reliable and valid measure that does not significantly increase the burden on respondents and has been used in a number of published studies, ${ }^{23-27}$ including studies conducted in China. ${ }^{24}$ In addition, participants were asked to compare their current sleep quality with that in the time before COVID-19 (response categories: $1=$ got worse, $2=$ no change, $3=$ got better). One item extracted from the PHQ-9 scale was used to assess whether participants were bothered by sleep disorder: 'Whether you have trouble falling or staying asleep, or sleeping too much in the past two weeks' (response categories: not at all, several days, more than half the days and nearly every day). ${ }^{6}$

\section{Pre-hospital factors}

Participants were asked to report whether they had received a diagnosis of insomnia or other chronic diseases (i.e., hypertension, diabetes mellitus, cancers, and other chronic heart/lung/liver/renal diseases) before COVID-19. Cigarette smoking within 1 year prior to COVID-19 was also recorded.

\section{In-hospital factors}

Information about severity level of COVID-19 at hospital admission, days in hospital, ICU admission, and the use of invasive ventilation and corticosteroid therapy was extracted from participants' medical records. Participants were asked whether they had a family member or members with COVID-19 or who had died of COVID19 , and whether they had witnessed the suffering and death of other COVID-19 patients. In addition, participants were asked whether they had received any mental health support services during their hospital stay.

\section{Post-hospital factors}

Participants reported whether they had received positive SARSCov-2 nucleic acid testing results and any mental health support services after hospital discharge.

Depressive symptoms were measured by a validated Chinese version of the PHQ-9 scale, which has been widely used for screening for depression in the Chinese population. ${ }^{24,28,29}$ The Cronbach's alpha of the PHQ-9 was 0.91 ; one factor was identified by exploratory factor analysis, explaining $59.1 \%$ of the total variance.

Generalised anxiety disorder was measured by a validated Chinese version of the Generalized Anxiety Disorder Scale (GAD7). ${ }^{30}$ The Cronbach's alpha of the GAD-7 Scale was 0.92; one factor was identified by exploratory factor analysis, explaining $68.1 \%$ of the total variance.

Post-traumatic stress disorder (PTSD) was measured by the eight-item Post-Traumatic Stress Disorder scale (PTSD-8). ${ }^{31}$ The items correspond to the DSM-IV criteria for PTSD. They are answered on a four-point Likert scale, ranging from 1 (not at all) to 5 (all the time). The summed score provides a score for symptom severity. The internal consistency as measured by Cronbach's alpha (0.89) was good in the current sample.

Four items were used to measure perceived emotional and instrumental support from family members and friends with a rating ranging from 0 (not at all) to 10 (very much). The Perceived Social Support Scale was formed by summing up individual item scores, with higher scores indicating perceived higher social support. The Cronbach's alpha of the Social Support Scale was 0.80;
Table 1 Sociodemographic characteristics of participants $(n=199)$

\begin{tabular}{|c|c|c|}
\hline & $n$ & $\%$ \\
\hline \multicolumn{3}{|l|}{ Age group (years) } \\
\hline $18-30$ & 33 & 16.6 \\
\hline $31-40$ & 59 & 29.6 \\
\hline $41-50$ & 35 & 17.6 \\
\hline $51-60$ & 33 & 16.6 \\
\hline$>60$ & 39 & 19.6 \\
\hline \multicolumn{3}{|l|}{ Gender } \\
\hline Male & 93 & 46.7 \\
\hline Female & 106 & 53.3 \\
\hline \multicolumn{3}{|l|}{ Relationship status } \\
\hline Currently single & 36 & 18.1 \\
\hline Married/cohabiting with a partner & 163 & 81.9 \\
\hline \multicolumn{3}{|l|}{ Highest education attained } \\
\hline Junior high or below & 53 & 26.6 \\
\hline Senior high & 57 & 28.6 \\
\hline College and above & 86 & 43.2 \\
\hline Refuse to disclose & 3 & 1.5 \\
\hline \multicolumn{3}{|l|}{ Permanent resident of the city } \\
\hline No & 146 & 73.4 \\
\hline Yes & 53 & 26.6 \\
\hline \multicolumn{3}{|l|}{ Monthly personal income (RMB) } \\
\hline No stable income & 71 & 35.7 \\
\hline$<3000$ & 25 & 12.6 \\
\hline $3000-5999$ & 52 & 26.1 \\
\hline 6000-9000 & 24 & 12.1 \\
\hline$\geq 10000$ & 27 & 13.6 \\
\hline \multicolumn{3}{|l|}{ Employment status } \\
\hline Full-time employment & 80 & 40.2 \\
\hline Freelancer & 31 & 16.1 \\
\hline Student & 15 & 7.5 \\
\hline Retiree & 55 & 27.6 \\
\hline Unemployed & 17 & 8.5 \\
\hline \multicolumn{3}{|l|}{ Having at least one child } \\
\hline No & 39 & 19.6 \\
\hline Yes & 160 & 80.4 \\
\hline \multicolumn{3}{|l|}{ City } \\
\hline Wuhan & 31 & 15.6 \\
\hline Shenzhen & 38 & 19.1 \\
\hline Dongguan & 35 & 17.6 \\
\hline Zhuhai & 39 & 19.6 \\
\hline Nanning & 56 & 28.1 \\
\hline
\end{tabular}

one factor was identified by exploratory factor analysis, explaining $62.8 \%$ of the total variance.

\section{Statistical analysis}

The score on the single-item SQS was used as the dependent variable. The associations between independent variables of interest (pre-hospital factors, in-hospital factors and post-hospital factors) and the dependent variable were tested using multivariate linear regression models, after adjusting for significant background characteristics. Adjusted unstandardised coefficients (B) and 95\% CIs were reported. SPSS version 24.0 (IBM Corporation) was used to conduct all analyses, with a $P$-value less than 0.05 indicating statistical significance. This was a secondary analysis of a study investigating behaviours and mental health of COVID-19 survivors in China.

\section{Results}

\section{Background characteristics}

Over half of the participants were aged 50 years or younger $(63.8 \%$, $n=127)$, female $(53.3 \%, n=106)$, were married or cohabiting with a partner $(81.9 \%, n=163)$, had not completed any tertiary education (55.3\%, $n=110)$, did not have permanent residency of the city where they lived $(73.4 \%, n=146)$, had a personal income of less 
Table 2 Sleep quality and its potential correlates among participants $(n=199)$

\begin{tabular}{|c|c|}
\hline & $n(\%)$ \\
\hline \multicolumn{2}{|l|}{ Sleep quality } \\
\hline \multicolumn{2}{|c|}{ Score on the single-item Sleep Quality Scale } \\
\hline Terrible (0) & $3(1.5)$ \\
\hline Poor (1-3) & $17(8.5)$ \\
\hline Fair (4-6) & $53(26.6)$ \\
\hline Good (7-9) & $108(54.2)$ \\
\hline Excellent (10) & $18(9.0)$ \\
\hline \multicolumn{2}{|c|}{$\begin{array}{l}\text { Whether you have trouble falling or staying asleep, or slee } \\
\text { in the past two weeks (an item on the PHQ-9) }\end{array}$} \\
\hline Not at all & $132(66.3)$ \\
\hline Several days & $39(19.6)$ \\
\hline More than half the days & $14(7.0)$ \\
\hline Nearly everyday & $14(7.0)$ \\
\hline
\end{tabular}

Self-reported change in sleep quality comparing current status with the time before COVID-19

$\begin{array}{lr}\text { Got worse } & 52(26.1) \\ \text { No change } & 145(72.9) \\ \text { Got better } & 2(1.0)\end{array}$

Pre-hospital factors

Self-reported insomnia diagnosis before CoVID-19

$\begin{array}{lc}\text { No } & 199(100.0) \\ \text { Yes } & 0(0.0)\end{array}$

Self-reported chronic conditions other than insomnia before COVID-19

No $132(66.3)$

$\begin{array}{ll}\text { Yes } & 67(33.7)\end{array}$

Smoking before COVID-19

No $177(55.3)$

Yes 22 (11.1)

In-hospital factors

Severity level of COVID-19 at hospital admission

Asymptomatic $3(1.5)$

Mild $\quad 42(21.1)$

Moderate $111(55.8)$

Severe $24(12.1)$

Critical $1(0.5)$

No record $18(9.0)$

Days in hospital

$\leq 14 \quad 22(11.1)$

$15-28 \quad 92(46.2)$

29-42 $49(24.6)$

$>42 \quad 11(5.5)$

No record $25(12.6)$

ICU admission
No
Yes

Yes $\quad 5(2.5)$

Invasive ventilation
No

Yes $\quad 7(3.5)$

Corticosteroid therapy

No $\quad 175(87.9)$

Yes 24 (12.1)

Witnessing the suffering of other COVID-19 patients

$\begin{array}{lr}\text { No } & 147(73.9) \\ \text { Yes } & 52(26.1)\end{array}$

Having a family member infected with COVID-19

No $110(55.3)$

Yes $\quad 89$ (44.7)

Having a family member die of COVID-19

No $196(98.5)$

Yes $3(1.5)$

Witnessing death of other COVID-19 patients

No $173(86.9)$

Yes 26 (13.1)

Receiving mental health support services during hospital stay

No $114(57.3)$

Yes $\quad 85$ (42.7)

Post-hospital factors

Receiving positive SARS-COV-2 nucleic acid testing results after hospital discharge

(Continued)

\section{Table 2 (Continued)}

\begin{tabular}{|c|c|c|}
\hline & $n(\%)$ & Mean (s.d.) \\
\hline $\begin{array}{l}\text { No/did not receive testing after hospital } \\
\text { discharge }\end{array}$ & $192(96.5)$ & \\
\hline Yes & $7(3.5)$ & \\
\hline \multicolumn{3}{|c|}{ Receiving mental health support services after hospital discharge } \\
\hline No & $155(77.9)$ & \\
\hline Yes & $44(22.1)$ & \\
\hline Depressive symptoms (score on $\mathrm{PHQ}-9)^{\mathrm{a}}$ & & $3.2(5.1)$ \\
\hline Anxiety symptoms (score on GAD-7) $)^{\mathrm{b}}$ & & $2.5(4.1)$ \\
\hline $\begin{array}{l}\text { Post-traumatic stress disorder (score on } \\
\text { PTSD-8) }\end{array}$ & & $28.5(9.6)$ \\
\hline Social Support Scale ${ }^{d}$ & & $11.7(5.5)$ \\
\hline \multicolumn{3}{|c|}{$\begin{array}{l}\text { a. PHQ-9, nine items, Cronbach's alpha: } 0.91 \text {; one factor was identified by exploratory } \\
\text { factor analysis, explaining } 59.1 \% \text { of total variance. } \\
\text { b. GAD-7, seven items, Cronbach's alpha: } 0.92 \text {; one factor was identified by exploratory } \\
\text { factor analysis, explaining } 68.1 \% \text { of total variance. } \\
\text { c. PTSD-8, eight items, Cronbach's alpha: } 0.89 \text {; one factor was identified by exploratory } \\
\text { factor analysis, explaining } 57.6 \% \text { of total variance. } \\
\text { d. Social Support Scale, four items, Cronbach's alpha: } 0.80 \text {; one factor was identified by } \\
\text { exploratory factor analysis, explaining } 62.8 \% \text { of total variance. }\end{array}$} \\
\hline
\end{tabular}

than RMB 6000 (approximately USD 900) per month (74.4\%, $n=$ 148), did not have a full-time job $(59.8 \%, n=119)$ and had at least one child $(80.4 \%, n=160)$ (Table 1$)$.

\section{Sleep quality}

Among the participants, $10.1 \%(n=20)$ reported terrible or poor sleep quality, and $26.6 \%(n=53)$ reported fair sleep quality. The mean score on the single-item SQS was 6.9 (s.d. 2.3). About 30\% of the participants $(26.1 \%, n=52)$ reported worse sleep quality when comparing their current status with the time before COVID-19. Over one-third of the participants had been bothered by a sleeping disorder in the past 2 weeks $(33.7 \%, n=67)$.

\section{Pre-hospital, in-hospital and post-hospital factors}

Prior to COVID-19, none of the participants had been diagnosed with insomnia, whereas $33.7 \%(n=67)$ had other chronic conditions. The most commonly reported chronic conditions were hypertension $(n=18,9.5 \%)$ and diabetes mellitus $(n=14,7.0 \%)$. Oneninth of the participants $(n=22,11.1 \%)$ had smoked in the year prior to COVID-19.

Based on their medical records, the majority of the participants had a moderate level of severity at hospital admission $(55.8 \%, n=$ $111)$, spent no more than 28 days in hospital $(57.3 \%, n=114)$, were not admitted to the ICU $(97.5 \%, n=194)$, and did not undergo invasive ventilation $(96.5 \%, n=192)$ or corticosteroid therapy $(87.9 \%, n=175)$. Among the participants, $26.1 \%(n=52)$ and $13.1 \%(n=26)$ had witnessed the suffering and death of other COVID-19 patients, $42.7 \%(n=85)$ had received mental health support during their hospital stay, $44.7 \%(n=89)$ reported having at least one family member infected with COVID-19 and 1.5\% $(n=3)$ had a family member who had died of COVID-19.

After hospital discharge, 3.5\% $(n=7)$ received positive SARSCov-2 nucleic acid testing results, and $22.1 \%(n=44)$ received mental health support services. The mean scores on the PHQ-9 scale, the GAD-7 scale, the PTSD-8 scale and the Social Support Scale are shown in Table 2.

\section{Factors associated with sleep quality}

Older participants (51-60 years: $\mathrm{B}=-1.12,95 \% \mathrm{CI}=-2.21,-0.03$, $P=0.04 ;>60$ years: $\mathrm{B}=-1.37,95 \% \mathrm{CI}=-2.42,-0.32, P=0.01$; reference group: $18-30$ years $)$ and those who were retired $(B=-1.20$, 95\% CI $=-1.97, \quad-0.42, \quad P=0.003$; reference group: full-time employment) reported poorer sleep quality. Participants who had a higher monthly income (RMB 3000-5999: $\mathrm{B}=0.83,95 \%$ 
Table 3 Correlates of sleep quality $(n=199)$

Unadjusted B1 $(95 \% \mathrm{Cl}) \quad P$-value

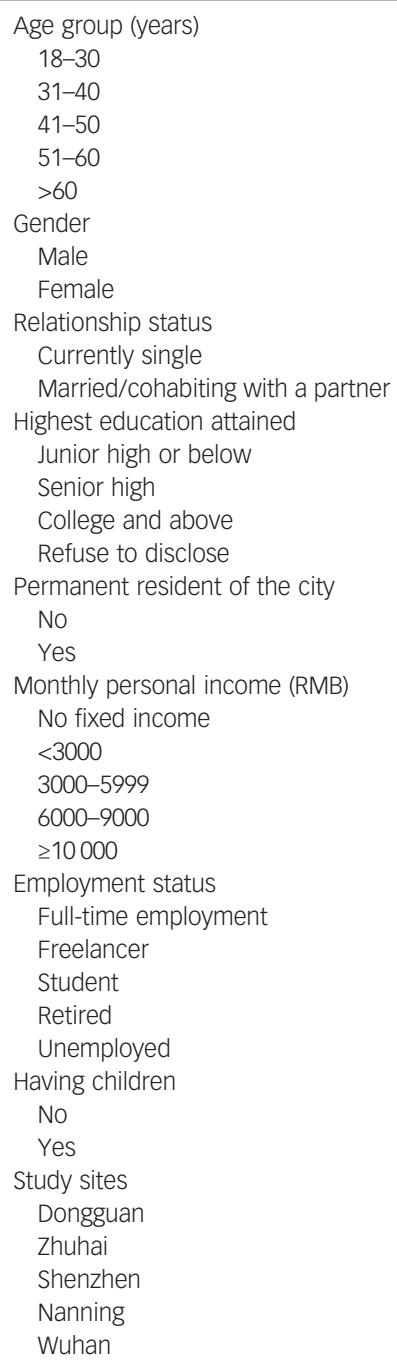

a. Unadjusted B: unstandardised coefficient.

$\mathrm{CI}=0.01,1.65, P=0.048$; reference group: no fixed income) and lived in Nanning $(\mathrm{B}=1.14,95 \% \mathrm{CI}=0.17,2.10, P=0.02$; reference group: Dongguan) reported better sleep quality (Table 3 ).

After controlling for these significant background characteristics, participants who had witnessed the suffering (adjusted $B=$ $-1.15,95 \% \mathrm{CI}=-1.70,-0.33, P=0.006$ ) or death (adjusted $\mathrm{B}=$ $-1.55,95 \% \mathrm{CI}=-2.62,-0.49, P=0.04)$ of other COVID-19 patients had poorer sleep quality. Regarding post-hospital factors, more severe depressive symptoms (adjusted $\mathrm{B}=-0.26,95 \%$ $\mathrm{CI}=-0.31, \quad-0.20, \quad P<0.001$ ), anxiety symptoms (adjusted $\mathrm{B}=-0.25,95 \% \mathrm{CI}=-0.33,-0.17, P<0.001$ ) and PTSD (adjusted $\mathrm{B}=-0.16,95 \% \mathrm{CI}=-0.22,-0.10)$ were associated with poorer sleep quality, whereas a higher level of social support (adjusted $\mathrm{B}=0.07,95 \% \mathrm{CI}=0.04,0.10, P<0.001)$ was associated with better sleep quality. The association between receiving positive SARSCov-2 nucleic acid testing results after hospital discharge and the dependent variable was of marginal statistical significance (adjusted $\mathrm{B}=-1.56,95 \% \mathrm{CI}=-3.36,0.24, P=0.09)$ (Table 4).

\section{Discussion}

In our study, the proportion of participants having had sleep disorders in the past 2 weeks (33.7\%) was slightly higher than that of a group of Chinese COVID-19 survivors 1 month after hospital discharge (29.2\%). ${ }^{6}$ Compared with a previous survey using the same one-item SQS to measure sleep quality among healthy Chinese factory workers in March 2020, the prevalence of terrible or poor sleep quality was much higher in our study $(10 \%$ v. $3.7 \%){ }^{24}$ These findings point to the necessity of interventions and services aimed at improving sleep quality among COVID-19 survivors.

The findings provide some empirical insights that could inform intervention development and service planning, and suggest the need to tailor such interventions and services to specific groups. Older participants were more likely to have poor sleep quality after hospital discharge. Previous studies have shown changes in the sleep cycles of elderly people, for instance, decreased total sleep time, increased time spent falling asleep at sleep onset, sleep fragmentation and daytime sleep. ${ }^{32}$ They should receive more attention in future programmes. Participants without a fixed income were more likely to have poor sleep quality. Although COVID-19 treatment is free in China, hospital admission and subsequent quarantine may affect the work of the survivors and may increase financial difficulties, especially among those with low or no fixed income. Financial difficulties represent a risk factor for sleep quality. ${ }^{33}$ Future programmes should consider providing financial assistance to COVID-19 survivors who have financial difficulties. Participants in Nanning reported better sleep quality than those in the other cities. Compared with the other four study sites, Nanning has been less affected by COVID-19. The COVID-19 situation in the city may be a structural factor influencing people's sleep quality.

In contrast to our hypothesis, pre-hospital factors such as presence of insomnia, other concurrent chronic conditions and smoking were not significantly associated with sleep quality in this study. Witnessing painful symptoms and deaths of other COVID-19 patients were significant in-hospital factors associated with poor sleep quality. These experiences are major psychological traumas for COVID-19 survivors, which may result in PTSD, a mental disorder leading to serious distress and disability. ${ }^{4,34}$ In line with other studies targeting COVID-19 survivors, ${ }^{7}$ our study found a high level of PTSD in this population. PTSD was negatively associated with sleep quality. ${ }^{12,35}$ Moreover, PTSD is long-lasting among survivors of infectious disease outbreaks (e.g., SARS). The median time taken for PTSD to remit is about 36 months for individuals who have sought mental health support services, and can be as long as 64 months for those who have never sought such services. ${ }^{36}$ A metaanalysis suggested that early intervention could lead to durable effects on PTSD severity. ${ }^{37}$ However, as the majority of participants had been discharged from hospitals before the government started providing mental health support services to all COVID-19 patients, ${ }^{14}$ only $42.7 \%$ received such services during their hospital stay. It is also unclear whether such mental health support services targeted traumatic experience, as the results showed a non-significant association between in-hospital mental health support service utilisation and sleep quality. In contrast to our hypothesis, ICU admission and the use of invasive ventilation and corticosteroids were not associated with post-discharge sleep quality, probably owing to the very small number of COVID-19 survivors who had such experiences (2.5-12.1\%).

Similar to previous findings, depressive symptoms and anxiety symptoms were significant post-hospital factors that were associated with poorer sleep quality. ${ }^{12}$ The associations between depressive and anxiety symptoms and sleep quality were bidirectional. Poor sleep quality might increase the risk of depression and anxiety, whereas presence of depressive symptoms or anxiety symptoms would increase the risk of sleep disturbance. ${ }^{12,38}$ One recent study suggested that sleep is a mediator of the relationship between stress and mental disorders in people infected with SARS-Cov-2. ${ }^{8}$ Such findings highlight the need to provide mental 


\begin{tabular}{|c|c|c|c|c|}
\hline & Unadjusted B (95\% Cl) & $P$-value & Adjusted B (95\% Cl) & $P$-value \\
\hline \multicolumn{5}{|l|}{ Pre-hospital factors } \\
\hline Presence of other chronic conditions before COVID-19 & $-0.36(-1.04,0.32)$ & 0.30 & $-0.09(-0.81,0.64)$ & 0.82 \\
\hline Smoking before COVID-19 & $0.44(-0.58,1.46)$ & 0.39 & $0.26(-0.78,1.30)$ & 0.62 \\
\hline \multicolumn{5}{|l|}{ In-hospital factors } \\
\hline \multicolumn{5}{|l|}{ Severity level of COVID-19 at hospital admission } \\
\hline Asymptomatic/mild & Ref & Ref & Ref & Ref \\
\hline Moderate & $-0.05(-0.81,0.80)$ & 0.99 & $-0.39(-1.30,0.51)$ & 0.39 \\
\hline Severe/critical & $-0.57(-1.71,0.55)$ & 0.32 & $-0.40(-1.66,0.85)$ & 0.53 \\
\hline No record & $0.25(-1.02,1.51)$ & 0.70 & $0.63(-0.64,1.91)$ & 0.33 \\
\hline \multicolumn{5}{|l|}{ Days in hospital } \\
\hline$\leq 14$ & Ref & Ref & Ref & Ref \\
\hline $15-28$ & $0.26(-0.82,1.34)$ & 0.64 & $0.69(-0.39,1.77)$ & 0.21 \\
\hline $29-42$ & $0.70(-0.47,1.87)$ & 0.24 & $0.77(-0.42,1.96)$ & 0.20 \\
\hline$>42$ & $0.46(-1.22 .2 .13)$ & 0.59 & $1.06(-0.64,2.77)$ & 0.22 \\
\hline No record & $0.50(-0.83,1.82)$ & 0.46 & $1.26(-0.09,2.60)$ & 0.07 \\
\hline ICU admission & $-1.56(-3.60,0.48)$ & 0.13 & $-1.43(-3.45,0.59)$ & 0.17 \\
\hline Invasive ventilation & $-1.25(-2.99,0.48)$ & 0.16 & $-1.01(-2.85,0.84)$ & 0.28 \\
\hline Corticosteroid therapy & $-0.82(-1.79,0.16)$ & 0.10 & $-0.82(-1.85,0.21)$ & 0.12 \\
\hline Having a family member infected with COVID-19 & $-0.17(-0.81,0.48)$ & 0.61 & $-0.37(-1.06,0.33)$ & 0.30 \\
\hline Having a family member die of COVID-19 & $0.75(-1.88,3.39)$ & 0.57 & $0.90(-1.76,3.56)$ & 0.51 \\
\hline Witnessing suffering of other COVID-19 patients & $-1.10(-1.81,-0.38)$ & 0.003 & $-1.15(-1.697,-0.33)$ & 0.006 \\
\hline Witnessing death of other COVID-19 patients & $-1.24(-2.18,-0.31)$ & 0.01 & $-1.55(-2.62,-0.49)$ & 0.04 \\
\hline Receiving mental health support services during hospital stay & $-0.30(-0.95,0.35)$ & 0.36 & $-0.25(-0.91,0.41)$ & 0.46 \\
\hline \multicolumn{5}{|l|}{ Post-hospital factors } \\
\hline Receiving positive SARS-Cov-2 nucleic acid testing results after hospital discharge & $-1.55(-3.28,0.18)$ & 0.08 & $-1.56(-3.36,0.24)$ & 0.09 \\
\hline Receiving mental health support services after hospital discharge & $0.48(-0.29,1.25)$ & 0.22 & $0.37(-0.43,1.18)$ & 0.37 \\
\hline Depressive symptoms (score on PHQ-9) & $-0.25(-0.31,-0.20)$ & $<0.001$ & $-0.26(-0.31,-0.20)$ & $<0.001$ \\
\hline Anxiety symptoms (score on GAD-7) & $-0.24(-0.32,-0.17)$ & $<0.001$ & $-0.25(-0.33,-0.17)$ & $<0.001$ \\
\hline Post-traumatic stress disorder (score on PTSD-8) & $-0.17(-0.22,-0.12)$ & $<0.001$ & $-0.16(-0.22,-0.10)$ & $<0.001$ \\
\hline Social Support Scale & $0.08(0.05,0.12)$ & $<0.001$ & $0.07(0.04,0.10)$ & $<0.001$ \\
\hline
\end{tabular}

health support services to COVID-19 survivors after hospital discharge. Currently, these mental health support services are provided to COVID-19 survivors upon their request, ${ }^{14}$ and utilisation is relatively low $(22.1 \%)$. Future programmes should actively reach out to COVID-19 survivors to increase coverage, and the services should be tailored to the needs of COVID-19 survivors. Increasing social support may be a useful strategy to improve post-discharge sleep quality. It is important to provide COVID-19-related health education to the general public and to family members of survivors, so as to reduce unrealistic fears and stigma towards COVID-19 survivors. These approaches may improve social support for COVID-19 survivors. In addition, having a positive SARS-Cov-2 retest result was marginally associated with poor sleep quality. As the cause of this phenomenon is still under investigation, ${ }^{19,20}$ receiving a positive retest result may trigger fear, worry and other negative emotional responses among COVID-19 survivors. In the future, longitudinal studies should be conducted to explore whether COVID-19 survivors with sleep disorders have worse recovery than those without.

This study had the strengths of recruiting survivors in multiple Chinese cities and considering pre-hospital, in-hospital and post-hospital factors. Although several studies have applied the single-item SQS in various Chinese populations, ${ }^{24,39}$ there has been no validation study conducted in a Chinese population. This was a weakness of this study. The study had some other limitations. First, there was no appropriate control group (e.g., patients who were admitted to hospital for reasons other than COVID-19 during the same period). Second, as compared with longer sleep questionnaires (e.g., PSQI), the single-item SQS was not able to cover important information such as sleep latency, sleep duration or habitual sleep efficiency. It merely reflected subjective sleep quality. Similar to some published cross-sectional studies, we measured self-reported changes in sleep quality before and after the COVID-19 outbreak. $^{6}$ However, such measurements are susceptible to strong recall bias. Therefore, we were not able to conclude that our participants had a decrease in sleep quality. Third, the sample size of this study was relatively small. The effect sizes of the non-significant associations were quite small, and the non-significance cannot be fully explained by the relatively small sample size. Fourth, COVID-19 survivors were recruited in five Chinese cities; generalisations should be made cautiously to other geographic locations in China. Fifth, we were not able to collect information from survivors who refused to participate in the study. Therefore, we could compare characteristics between participants and those who refused to complete the survey and hence could not estimate the impact of refusals on study outcomes. Selection bias existed. Sixth, parts of the data were selfreported, and verification was not feasible. Recall bias may have occurred, and we were not able to estimate the impact of recall bias on study outcomes. Moreover, we did not collect information about history of psychiatric disorders, which is a significant factor in post-discharge sleep quality. Furthermore, this was a cross-sectional study and could not establish causal relationships.

Among the COVID-19 survivors, a significant proportion were bothered by sleep disorders, and many reported terrible or poor sleep quality 6 months after hospital discharge. Witnessing the suffering or death of other COVID-19 patients was a significant in-hospital factor associated with poor sleep quality after hospital discharge. Depressive symptoms, anxiety symptoms, PTSD and social support were significant post-hospital factors influencing sleep quality. Interventions and support services to improve sleep quality should be provided to COVID-19 survivors. 
Leiwen Fu (D), School of Public Health (Shenzhen), Sun Yat-sen University, Shenzhen, China; Yuan Fang, Department of Early Childhood Education, The Education University of Hong Kong, Hong Kong SAR, China; Dan Luo, School of Public Health (Shenzhen), Sun Yat-sen University, Shenzhen, China; Bingyi Wang (D), School of Public Health (Shenzhen), Sun Yat-sen University, Shenzhen, China; Xin Xiao, School of Public Health (Shenzhen), Sun Yat-sen University, Shenzhen, China, and Center for Optometry and Visual Science, the People's Hospital of Guangxi Zhuang Autonomous Region, Nanning China; Yuqing Hu, School of Public Health (Shenzhen), Sun Yat-sen University, Shenzhen, China; Niu Ju, School of Public Health (Shenzhen), Sun Yat-sen University, Shenzhen, China; Weiran Zheng, School of Public Health (Shenzhen), Sun Yat-sen University, Shenzhen, China; Hui Xu, School of Public Health (Shenzhen), Sun Yat-sen University, Shenzhen, China; Xue Yang, JC School of Public Health and Primary Care, Faculty of Medicine, The Chinese University of Hong Kong, Hong Kong SAR, China; Pau Shing Fong Chan, IC School of Public Health and Primary Care, Faculty of Medicine, The Chinese University of Hong Kong Hong Kong SAR, China: Zhijie Xu, The Fifth Affiliated Hospital of Sun Yat-sen University, Zhuhai, China; Ping Chen, The Fifth Affiliated Hospita of Sun Yat-sen University, Zhuhai, China; Jiaoling He, The Fifth Affiliated Hospital of Sun Yat-sen University, Zhuhai, China; Hongqiong Zhu, The Fifth Affiliated Hospital of Sun Yat-sen University, Zhuhai, China; Huiwen Tang, The Fifth Affiliated Hospital of Sun Yatsen University, Zhuhai, China; Dixi Huang, The Fifth Affiliated Hospital of Sun Yat-sen University, Zhuhai, China; Zhongsi Hong, The Fifth Affiliated Hospital of Sun Yat-sen University, Zhuhai, China: Xiaojun Ma, Guangdong Provincial People's Hospital, Guangzhou, China; Yanrong Hao, Department of Scientific Research, The People's Hospital of Guangxi Zhuang Autonomous Region, Nanning, China; Lianying Cai, Department of Education, The People's Hospital of Guangxi Zhuang Autonomous Region Nanning, China; Jianrong Yang, Department of Hepatobiliary, Pancreas and Spleen Surgery, The People's Hospital of Guangxi Zhuang Autonomous Region, Nanning, China; Shupei Ye, Department of emergency, SSL Central Hospital of Dongguan City,

Dongguan, China: Jianhui Yuan, Shenzhen Nanshan District Center for Disease Contro and Prevention, Shenzhen, China; Yao-Qing Chen, School of Public Health (Shenzhen), Sun Yat-sen University, Shenzhen, China; Fei Xiao, The Fifth Affiliated Hospital of Sun Yat-sen University, Zhuhai, China; Zixin Wang (D), JC School of Public Health and Primary Care, Faculty of Medicine, The Chinese University of Hong Kong, Hong Kong SAR, China; Huachun Zou, School of Public Health (Shenzhen), Sun Yat-sen University, Shenzhen, China; Kirby Institute, University of New South Wales, Sydney, Australia; Shenzhen Cente for Disease Control and Prevention, Shenzhen, China; and School of Public Health, Shanghai Jiao Tong University, Shanghai, China

Correspondence: Huachun Zou. Email: Zouhuachun@mail.sysu.edu.cn

First received 16 Dec 2020, final revision 11 Aug 2021, accepted 30 Aug 2021

\section{Data availability}

The data that support the findings of this study are available from the corresponding author, H.Z., upon reasonable request.

\section{Acknowledgements}

We thank Dr Yusheng Jie from the Third Affiliated Hospital of Sun Yat-sen University for his support.

\section{Author contributions}

H.Z., Z.W. and F.X. conceived the study and designed the protocol with L.F. and Y.F. L.F., Y.F. D.L. and B.W. conducted this study. L.F., Y.F., D.L. and B.W. contributed to statistical analysis and interpretation of data. L.F., Y.F., D.L., B.W., F.X., Z.W. and H.Z. drafted the manuscript with all authors critically revising the manuscript. All authors have read and approved the final version of the manuscript.

\section{Funding}

This study was supported by the Natural Science Foundation of China Excellent Young Scientists Fund (82022064), Natural Science Foundation of China International/Regional Research Collaboration Project (72061137001), Natural Science Foundation of China Young Scientist Fund (81703278), the Australian National Health and Medical Research Commission Early Career Fellowship (APP1092621), the National Science and Technology Major Project of China (20187X10721102), the Sanming Project of Medicine in Shenzhen (SZSM201811071), the High Level Project of Medicine in Longhua, Shenzhen (HLPM201907020105), the National Key Research and Development Program of China (2020YFC0840900) and the Guangxi Medical and Health Appropriate Technology Development and Application Project (S2020124). None of the funding parties had any role in the design of the study or in the explanation of the data.

\section{Declaration of interest}

None.

\section{References}

1 Magnavita N, Sleep GS. Health and wellness at work: a scoping review. Int J Environ Res Public Health 2017; 14(11): 1347.

2 Grandner MA, Sands-Lincoln MR, Pak VM, Garland SN. Sleep duration, cardiovascular disease, and proinflammatory biomarkers. Nat Sci Sleep 2013; 5: 93-107.

3 Li L, Wu C, Gan Y, Qu X, Lu Z. Insomnia and the risk of depression: a metaanalysis of prospective cohort studies. BMC Psychiatry 2016; 16(1): 375.
4 Liu D, Baumeister RF, Veilleux JC, Chen C, Liu W, Yue Y, et al. Risk factors associated with mental illness in hospital discharged patients infected with COVID19 in Wuhan, China. Psychiatry Res 2020; 292: 113297.

5 Holmes EA, O'Connor RC, Perry VH, Tracey I, Wessely S, Arseneault L, et al. Multidisciplinary research priorities for the COVID-19 pandemic: a call for action for mental health science. Lancet Psychiatry 2020; 7(6): 547-60.

6 Wu C, Hu X, Song J, Yang D, Xu J, Cheng K, et al. Mental health status and related influencing factors of COVID-19 survivors in Wuhan, China. Clin Transl Med 2020; 10(2): e52.

7 Mazza MG, De Lorenzo R, Conte C, Poletti S, Vai B, Bollettini I, et al. Anxiety and depression in COVID-19 survivors: role of inflammatory and clinical predictors. Brain Behav Immun 2020; 89: 594-600.

8 Magnavita N, Tripepi G, Di Prinzio RR. Symptoms in health care workers during the COVID-19 epidemic. a cross-sectional survey. Int J Environ Res Public Health 2020; 17(14): 5218

9 Bani-Sadr F, Hentzien M, Pascard M, N'Guyen Y, Servettaz A, Andreoletti L, et al. Corticosteroid therapy for patients with COVID-19 pneumonia: a beforeafter study. Int J Antimicrob Agents 2020; 56(2): 106077.

10 Huang C, Huang L, Wang Y, Li X, Ren L, Gu X, et al. 6-month consequences of COVID-19 in patients discharged from hospital: a cohort study. Lancet 2021; 397(10270): 220-32

11 Tansey CM, Louie M, Loeb M, Gold WL, Muller MP, de Jager J, et al. One-year outcomes and health care utilization in survivors of severe acute respiratory syndrome. Arch Intern Med 2007; 167(12): 1312-20.

12 Altman MT, Knauert MP, Pisani MA. Sleep disturbance after hospitalization and critical illness: a systematic review. Ann Am Thorac Soc 2017; 14(9): 1457-68.

13 Cole JL. Steroid-induced sleep disturbance and delirium: a focused review for critically III patients. Fed Pract 2020; 37(6): 260-7.

14 Government of People's Republic of China. Plans to Provide Psychological Support and Social Services for COVID-19 Patients and their Family Members, and People under Quarantine. 2020 (http://www.gov.cn/xinwen/ 2020-04/08/content_5500131.htm [cited 18 Nov 2020]).

15 Yang $\mathrm{X}$, Yang X, Kumar $\mathrm{P}, \mathrm{Cao} B$, Ma X, Li T. Social support and clinical improvement in COVID-19 positive patients in China. Nurs Outlook 2020; 68(6): 830-7.

16 Cao H, Ruan L, Liu J, Liao W. The clinical characteristic of eight patients of COVID-19 with positive RT-PCR test after discharge. J Med Virol 2020; 92(10): 2159-64.

17 Hu F, Chen F, Ou Z, Fan Q, Tan X, Wang Y, et al. A compromised specific humoral immune response against the SARS-CoV-2 receptor-binding domain is related to viral persistence and periodic shedding in the gastrointestinal tract. Cell Mol Immunol 2020; 17(11): 1119-25.

18 Shui TJ, Li C, Liu HB, Chen X, Zhang BK. Characteristics of recovered COVID-19 patients with recurrent positive RT-PCR findings in Wuhan, China: a retrospective study. BMC Infect Dis 2020; 20(1): 749.

19 Tao W, Wang X, Zhang G, Guo M, Ma H, Zhao D, et al. Re-detectable positive SARS-CoV-2 RNA tests in patients who recovered from COVID-19 with intestinal infection. Protein Cell 2020; 12(3): 230-5.

20 Wu F, Zhang W, Zhang L, Wang D, Wan Y. Discontinuation of antiviral drugs may be the reason for recovered COVID-19 patients testing positive again. Br J Hosp Med 2020; 81(4): 1-2

21 National Health Commission of The People's Republic of China. Update of the COVID-19 Pandemic in China (November 9, 2020). Available from: http:// www.nhc.gov.cn/xcs/yqfkdt/202011/663e3753744f41d0b506324fa719d1be. shtml [cited 10 Nov 2020].

22 Snyder E, Cai B, DeMuro C, Morrison MF, Ball W. A new single-item sleep quality scale: results of psychometric evaluation in patients with chronic primary insomnia and depression. J Clin Sleep Med 2018; 14(11): 1849-57.

23 Serlachius A, Schache K, Boggiss A, Lim D, Wallace-Boyd K, Brenton-Peters J, et al. Coping skills mobile app to support the emotional well-being of young people during the COVID-19 pandemic: protocol for a mixed methods study. Res Protoc 2020; 9(10): e23716.

24 Pan $Y$, Xin M, Zhang C, Dong W, Fang Y, Wu W, et al. Associations between COVID-19 specific information exposure, mental health and self-reported compliance with personal preventive measures: evidence from a cross-sectional study at the initial phase of work resumption following the COVID-19 outbreak in China J Med Internet Res 2020; 22(10): 222596.

25 Gupta R, Grover S, Basu A, Krishnan V, Tripathi A, Subramanyam A, et al Changes in sleep pattern and sleep quality during COVID-19 lockdown. Indian J Psychiatry 2020; 62(4): 370-8.

26 Badahdah A, Khamis F, Al Mahyijari N, Al Balushi M, Al Hatmi H, Al Salmi I, et al. The mental health of health care workers in Oman during the COVID-19 pandemic. Int J Soc Psychiatry 2020; 67(1): 90-5.

27 Shrestha C, Ghimire C, Acharya S, Prabhat KC, Singh S, Sharma P. Mental wellbeing during the lockdown period following the COVID-19 pandemic in Nepal: a descriptive cross-sectional study. J Nepal Med Assoc 2020; 58(230): $744-50$. 
28 Wang W, Bian Q, Zhao Y, Li X, Wang W, Du J, et al. Reliability and validity of the Chinese version of the Patient Health Questionnaire (PHQ-9) in the general population. Gen Hosp Psychiatry 2014; 36(5): 539-44.

29 Pan Y, Fang Y, Xin M, Dong W, Zhou L, Hou Q, et al. Self-reported compliance with personal preventive measures among 3035 Chinese factory workers at the beginning of work resumption following COVID-19 outbreak: a cross-sectional online survey. J Med Internet Res 2020; 22(9): e22457.

30 Tong X, An D, McGonigal A, Park SP, Zhou D. Validation of the Generalized Anxiety Disorder-7 (GAD-7) among Chinese people with epilepsy. Epilepsy Res 2016; 120: 31-6.

31 Hansen M, Andersen TE, Armour C, Elklit A, Palic S, Mackrill T. PTSD-8: a short PTSD inventory. Clin Pract Epidemol Ment Health 2010; 6: 101.

32 Chen CJ, Hsu LN, McHugh G, Campbell M, Tzeng YL. Predictors of sleep quality and successful weaning from mechanical ventilation among patients in respiratory care centers. J Nurs Res 2015; 23(1): 65-74.

33 Hall M, Buysse DJ, Nofzinger EA, Reynolds CF 3rd, Thompson W, Mazumdar S, et al. Financial strain is a significant correlate of sleep continuity disturbances in late-life. Biol Psychol 2008; 77(2): 217-22.

34 Xiao S, Luo D, Xiao Y. Survivors of COVID-19 are at high risk of posttraumatic stress disorder. Glob Health Res Policy 2020; 5: 29.
35 Mantua J, Helms SM, Weymann KB, Capaldi VF 2nd, Lim MM. Sleep quality and emotion regulation interact to predict anxiety in veterans with PTSD. Behav Neurol 2018; 2018: 7940832

36 Kessler RC, Chiu WT, Demler O, Merikangas KR, Walters EE. Prevalence, severity, and comorbidity of 12-month DSM-IV disorders in the National Comorbidity Survey Replication. Arch Gen Psychiatry 2005; 62(6): 617-27.

37 Oosterbaan V, Covers MLV, Bicanic IAE, Huntjens RJC, de Jongh A. Do early interventions prevent PTSD? A systematic review and meta-analysis of the safety and efficacy of early interventions after sexual assault. Eur $J$ Psychotraumatol 2019; 10(1): 1682932.

38 Orwelius L, Nordlund A, Nordlund P, Edéll-Gustafsson U, Sjöberg F. Prevalence of sleep disturbances and long-term reduced health-related quality of life after critical care: a prospective multicenter cohort study. Crit Care 2008; 12(4): R97.

39 Zhao Y, Song J, Brytek-Matera A, Zhang H, He J. The relationships between sleep and mental and physical health of Chinese elderly: exploring the mediating roles of diet and physical activity. Nutrients 2021; 13(4): 1316. 